\title{
On Attentional Control as a Source of Residual Shift Costs: Evidence From Two-Component Task Shifts
}

\author{
Ronald Hübner \\ Universität Konstanz
}

\author{
Torsten Futterer \\ Technische Universität Braunschweig
}

\author{
Marco Steinhauser \\ Universität Konstanz
}

\begin{abstract}
It is widely assumed that supervisory or attentional control plays a role only in the preparatory reconfiguration of the mental system in task shifting. The well-known fact that residual shift costs are still present even after extensive preparation is usually attributed to passive mechanisms such as cross talk. The authors question this view and suggest that attentional control is also responsible for residual shift costs. The authors hypothesize that, under shift conditions, tasks are executed in a controlled mode to guarantee reliable performance. Consequently, the control of 2 task components should require more resources than the control of only 1 . A series of 4 experiments with 2 -component tasks was conducted to test this hypothesis. As expected, more residual shift costs were observed when 2 components rather than 1 varied across trials. Interference effects and sequential effects could not account for these results.
\end{abstract}

\begin{abstract}
Although much research effort has been put forth during the last few decades in investigating mental control (for overviews see, e.g., Egeth \& Yantis, 1997; Johnston \& Dark, 1986; Kinchla, 1992), the specific mechanisms and the interactions between stimulus triggered processes and voluntary control are still largely unknown. This holds as well for higher executive mechanisms that coordinate and control shifting between different tasks (e.g. Allport, Styles, \& Hsieh 1994; Logan, 1985; Meiran, 1996; Monsell, 1996; Norman \& Shallice, 1986; Rogers \& Monsell, 1995; Shallice, 1994) and that are the topic of the present article. Nevertheless, several promising concepts have been proposed (cf. Baddeley, 1986; Norman \& Shallice, 1986; Shallice \& Burgess, 1996; Shiffrin \& Schneider, 1977). For instance, Norman and Shallice (1986) developed, from a neuropsychology perspective, a widely acknowledged model in which it is assumed that external stimuli automatically activate well-learned action schemas. When several schemas compete for selection, a so-called contention-scheduling mechanism resolves this conflict. However, in situations in which a goal-specific schema must be selected against strong competition, contention scheduling is not sufficient, and voluntary or endogenous control is necessary. It is assumed that this type of control is exerted by a supervisory attentional system (SAS) that affects the selection of schemas indirectly by biasing their activation.
\end{abstract}

Ronald Hübner and Marco Steinhauser, Fachbereich Psychologie, Universität Konstanz, Konstanz, Germany; Torsten Futterer, Institut für Psychologie, Technische Universität Braunschweig, Braunschweig, Germany.

Correspondence concerning this article should be addressed to Ronald Hübner, Universität Konstanz, Fachbereich Psychologie, Fach D29, D-78457 Konstanz, Germany. Electronic mail may be sent to Ronald.Huebner@uni-konstanz.de.
The SAS and similar concepts assume that supervisory control is accomplished by a unitary system of limited capacity that operates serially. One experimental paradigm that seems suited for investigating such issues is task shifting (e.g. Jersild,1927; Rogers \& Monsell, 1995; Spector \& Biederman, 1976), which has been considered as the prototype of situations requiring endogenous control (e.g. Logan, 1985; Meiran, 1996). In respective studies, participants must alternate between different tasks across trials. Usually, this produces costs with respect to response times and errors relative to nonalternate conditions.

Recently, however, Allport et al. (1994) questioned whether a unitary supervisory control mechanism is involved in task shifting. They derived and tested two predictions. First, when supervisory control is capacity limited, controlling two components of a task should require more resources than controlling only one component. Second, the control of difficult tasks should consume more capacity than the control of easier ones. Although most task-shift studies have applied simple tasks, Allport et al. had to use tasks with two components to test their first prediction. Their participants worked through lists of alternating tasks or stimulus dimensions. Odd-even and less-greater judgments served as tasks, whereas group size and the value of a set of identical numerals served as dimensions. First of all, Allport et al. observed that shifting between tasks produced about the same costs as shifting between stimulus dimensions. However, concurrent shifts between both tasks and dimensions produced no extra costs relative to shifts of a single component. This result seemed to contradict their first prediction.

With respect to the second prediction, Allport et al. (1994) examined shifts between difficult and less difficult tasks. For instance, they varied task difficulty by manipulating the symbolic distance in numeric comparisons. As a result, task-shift costs did not depend on task difficulty, which was regarded as incompatible 
with the assumption of a capacity limited control system. Allport et al. admitted that the result could also be explained by a stagelike process in which mental control first shifts between tasks and then allocates its capacity to task execution. However, they rejected this alternative account, because the assumption that mental control operates strictly serially contradicted their result that concurrent shifts produced the same amount of costs as shifts of a single component. Thus, Allport et al. concluded that a unitary control system is not involved in task shifting.

Furthermore, Allport et al. (1994) observed that even considerably prolonged intertrial intervals could not prevent shift costs. From this, they inferred that the costs usually observed in task-shift studies were not due to a task-shift stage that had to be completed before execution. Rather, they proposed that the costs were caused by proactive interference. That is, the stimulus - response (S-R) mappings of what they called the "old instruction set" are still active after a shift and compete with the mappings of the actual task. This competition produces interference and, consequently, slows responses. Because Allport et al. found that the proactive interference from the old task dissipates relatively slowly and gradually over several trials, they called this phenomenon task-set inertia.

The results of Allport et al. (1994) are rather disappointing for someone who hopes to gain insight into supervisory control mechanisms from task-shift studies. However, strictly speaking, Allport et al. described the consequences of task-set shifting rather than its mechanisms. Moreover, we show in the present article that their conclusions are not justified, because they considered only a restricted set of experimental conditions. Furthermore, Allport et al.'s account is rather vague. For instance, it remains unclear exactly how and where proactive interference takes place. The assumption that it is caused by competing S-R mappings seems inappropriate for explaining shift costs. For instance, when stimuli are compatible - that is, both trigger the same response - they should facilitate responses or, at least, not impede them. However, shift costs occur also for compatible stimuli.

This discrepancy has been resolved by Rogers and Monsell (1995), who investigated shift costs by examining sequential effects. Their participants had to work through different tasks that were presented as alternating runs. Shift costs were defined as the performance reduction on shift trials relative to nonshift trials. The fact that considerable shift costs were also observed for responsecompatible stimuli led Rogers and Monsell to distinguish between cross talk and task cuing. Cross talk is defined as interference between S-R mappings, whereas task cuing refers to interference between task sets or control schemas. In principle, both types of interference can be more or less proactive and related to the inertia of corresponding processes. However, unlike Allport et al. (1994), Rogers and Monsell found that performance improved only between the first and second trials of a run, which they regarded as incompatible task-set with inertia. Nevertheless, their experiments also showed that shift costs could not be eliminated by increasing the intertrial interval.

To account for their results, Rogers and Monsell (1995) proposed a two-component model of task-set shifting. One component comprises endogenous control, which partly reconfigures the cognitive system according to the new task. The other component consists of stimulus-triggered processes (i.e., exogenous control,) that inhibit processes previously activated for executing the old task and complete the reconfiguration. The latter part of the model is called the stimulus-cued completion hypothesis. Thus, contrary to Allport et al. (1994), Rogers and Monsell assumed a prestimulus control stage in which the mental system is partly reconfigured before task execution. Because this stage is not sufficient for explaining shift costs, they assumed that the residual portion of the costs is due to stimulus-driven control processes, whose duration depends on the amount of proactive interference; however, this interference is caused not only by competing $S-R$ mappings (cross talk) but also by competing task sets (task cuing).

A similar account has recently been proposed by Rubinstein, Meyer, and Evans (in press). They used a production-system architecture and also assumed two stages: goal shifting and rule activation. Goal shifting can occur in the preparation phase, whereas rule activation takes place after stimulus identification.

Taken together, these results suggest that it is appropriate to differentiate between two different types of control processes involved in task shifting (see also Meiran, 1996). The first type relates to preparation and anticipatory reconfiguration of the mental system with respect to the new task, whereas the second type concerns control that takes place after the arrival of the new stimulus. According to Rogers and Monsell (1995), the latter control type is necessary to resolve the conflict between the old and new task sets or to complete the reconfiguration of the mental system. Similarly, Rubinstein et al. (in press) assumed that stimulus features help to activate the appropriate rules and deactivate the old ones.

An important question is whether both control types are supervisory processes. Some researchers sort exclusively the first type into this category (e.g., Meiran, 1996; Rogers \& Monsell, 1995). For instance, Meiran (1996) proposed two necessary criteria for identifying supervisory control processes: First, they must be specifically related to the process of task-set shifting, and, second, control commands must precede task execution. Therefore, these researchers focus mainly on costs for anticipatory reconfiguration and show little interest in residual shift costs. However, it is questionable that this restricted view is appropriate. For instance, it is conceivable that supervisory control also takes place during task execution. Indeed, it has widely been accepted that such control is required during the execution of novel tasks. Why should this not hold also for execution after a task shift? This question is examined in the present article. We report a series of experiments in which we investigated the idea that residual shift costs reflect, at least partly, the involvement of supervisory control. Specifically, we tested the attentional control hypothesis (AC hypothesis), which is based on the "controlled processing" approach of Shiffrin and Schneider (1977; Schneider \& Shiffrin, 1977) and according to which task execution requires more attentional control under shift conditions than under nonshift conditions.

As did Allport et al. (1994), we used two-component tasks and predicted that more attentional control is necessary when both components have to be shifted than when one has to be shifted. At first glance, this seems to contradict the results of Allport et al, who found no extra costs for concurrent shifts of tasks and stimulus dimensions. However, the generality of their results is restricted, because in their experiment each task was uniquely linked to a specific stimulus dimension. Here we provide data indicating that the results are different when tasks and dimensions vary independently. 
Again following Allport et al. (1994), we investigated concurrent shifts between stimulus dimensions and tasks; tasks were odd-even and less-greater judgments. However, instead of group size and the value of a group of numerals, the global and local levels of compound numbers (see Figure 1) served as stimulus dimensions. It is known that shifts between the levels of such hierarchical stimuli produce costs (e.g. Hübner, 1997, 2000; Robertson, 1996; Ward, 1982). Thus, in our experiments the participants had to extract a numeral from the prespecified level and classify it according to the required task.

To make the possibly involved mental structures and pathways more explicit, we designed a theoretical framework with respect to our tasks. One method would have been to use a productionsystem architecture as, for instance, in Rubinstein et al. (in press). However, we preferred a network structure that bears close resemblance to connectionist models (e.g., Cohen, Dunbar, \& McClelland, 1990). The outline of our framework can be seen in Figure 2. Each combination of level and task corresponds to a certain pathway, considered as a specific S-R mapping. Consequently, a stimulus that activates more than one pathway causes cross talk. Furthermore, without endogenous control the response is determined by the strongest pathway. Thus, to allow goal-directed behavior, control units were added that biased the activation in the task-relevant pathway. This type of control was adopted from Cohen et al. (1990). Although details of the model have been criticized (Kanne, Balota, Spieler, \& Faust, 1998; Mewhort, Braun, \& Heathcote, 1992), two aspects are particularly important for our objective. First, the activation of the control nodes (i.e., effort), is maintained throughout task execution. Second, the need for control is not all or none. Rather, Cohen et al. emphasized that tasks reside along a continuum between automatic and controlled.

How can shift costs be explained in our framework? One possibility would be to assume that task shifts increase the cross talk between the competing pathways (S-R mappings) and, consequently, the response times as well (cf. Allport et al., 1994). On the other hand, according to Rogers and Monsell (1995), a stimulus also activates irrelevant control units on shift trials, which produces costs. To enable such task cuing, one would have to add connections between the input and control units. In a modification of the Cohen et al. model, Cohen and Huston (1994) implemented such connections as well as connections between the control units. Although we assume similar connections in our framework, they were, for simplicity, omitted in Figure 2.

Finally, a third explanation is given by our AC hypothesis, according to which attentional control effort during task execution

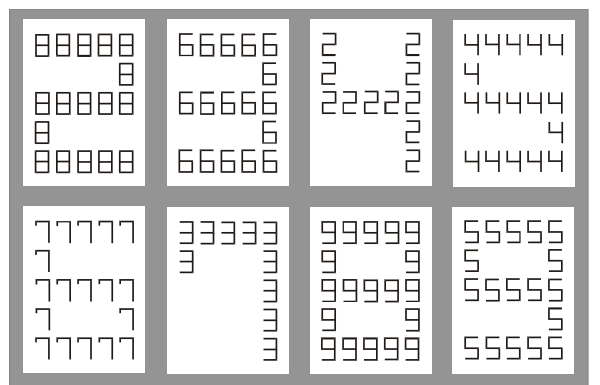

Figure 1. Examples of the compound numerals used as stimuli in the experiments.

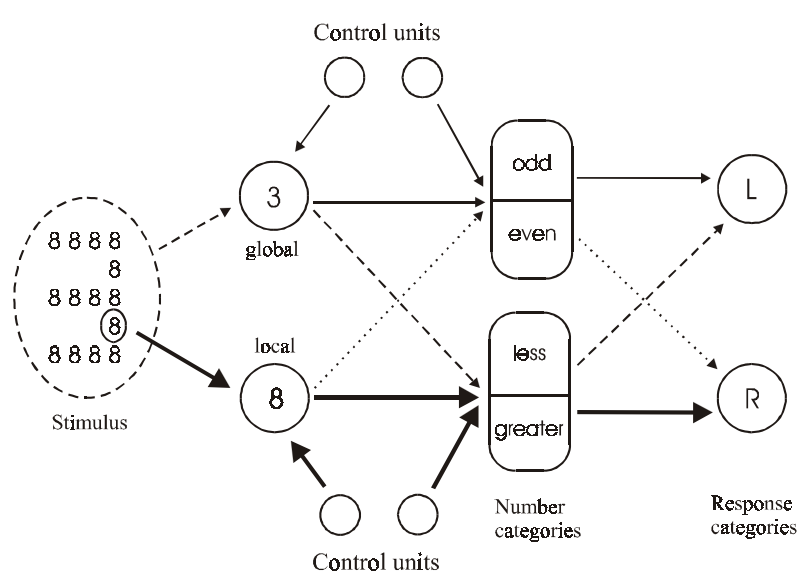

Figure 2. The structure of our tasks and possible involved processing stages and pathways. The different arrow types represent the flow of activation for specific examples (see text).

is increased under task shifting. As suggested by our framework, we assume that task execution proceeds sequentially. First, the numeral at the relevant level is extracted, and then the relevant task is selected and performed. We further assume that each of these selection or decision steps requires more or less attentional control. More control is required when the selections vary across trials, such as, when the local numeral has to be selected on some trials and the global one on others. Additional control is necessary when there is cross talk, that is, competition from nonselected information. In any case, the amount of attentional control is adjusted so that the mental system is capable of executing the current task reliably.

This account can also easily explain why there are no extra shift costs for concurrent shifts when dimensions and tasks are uniquely linked. Because the selected level determines the relevant task, the corresponding control nodes are strongly associated. Therefore, activating the control node for the selection of the relevant level also activates the associated task control node. Consequently, task selection occurs automatically with level selection and requires no extra control. In other words, only one decision step is required in conditions with uniquely linked levels and tasks. It follows from this explanation that task selection requires an additional controlled selection step when levels and tasks vary independently across trials. Accordingly, residual shift costs should increase. This prediction was tested in Experiment 1.

\section{Experiment 1}

The first experiment was conducted to test the hypothesis that there are extra residual costs for shifts between independent task components relative to conditions with one variable component and two linked components. Therefore, in addition to the conditions used by Allport et al. (1994), we included blocks of trials in which tasks and levels varied independently. Furthermore, we used a slightly different procedure. In the experiments of Allport et al., the participants had to work through lists of alternating tasks in a self paced manner. They had to respond verbally, and the dependent variable was list completion time. Therefore, costs for prep- 
aration and those for execution were mixed. Here the participants had to respond manually, and the latencies for the individual stimuli were registered. Moreover, in shift conditions, tasks or dimensions (or both) were randomized within a block of trials and indicated by cues. To separate preparation from execution, the participants could inspect the cues as long as they wanted before the next stimulus was presented. The duration of this interval, labeled "preparation time", was registered. Our approach is similar to that of Dixon (1981) and Dixon and Just 1986). They assumed that such an interval reflects the time required for selecting an attention algorithm.

\section{Method}

\section{Participants}

Ten participants ( 5 male and 5 female), who ranged in age from 23 to 39 years, took part in the experiment. All were right-handed (by self report) and had normal or corrected-to-normal vision.

\section{Apparatus}

The stimuli were presented on a 19 -in. $(48-\mathrm{cm})$ color monitor connected to a personal computer that also controlled stimulus presentation and response registration.

\section{Stimuli}

Stimuli were compound numerals whose global shape was constructed from identical local numerals in a $5 \times 5$ grid. At a viewing distance of 127 $\mathrm{cm}$, the global numerals extended a visual angle of $1.71^{\circ}$ horizontally and of $2.34^{\circ}$ vertically, and the local numerals extended $0.23^{\circ}$ and $0.34^{\circ}$, respectively. The stimuli were white $(82 \mathrm{~cd} / \mathrm{m} 2)$ on a black $\left(0.314 \mathrm{~cd} / \mathrm{m}^{2}\right)$ background. Exa mples of the stimuli can be seen in Figure 1. The numerals ranged from 2 to 9 . When all values for the two target levels were combined, there were 64 different stimuli.

\section{Procedure}

The participants had to decide whether the numeral at the given target level was odd or even, or whether it was less than or equal to 5,or greater than or equal to 6 , and they had to respond by pressing one of two response buttons with the index or middle finger of the same hand. Mapping of the response categories to the keys (or fingers) was counterbalanced across participants. Each trial began with the appearance of a cue that was centered on the screen and could have one of two forms and one of two sizes. The odd-even task was indicated by an ellipse and the less-greater task by a square, whereas the target level was indicated by the size of the cue, which corresponded to that of the global stimulus shape or to that of one local element. For instance, a small ellipse indicated that the odd-even task had to be performed with the value of the local numerals.

After the participants had initiated the trial by pressing a start key with the hand that was not used for responding to the target stimuli, a blank screen appeared for $400-500 \mathrm{~ms}$, followed by the stimulus, which was exposed randomly $1^{\circ}$ (measured to the center of the stimulus) left or right of the fixation point for $133 \mathrm{~ms}$. The cue for the next trial appeared 1,000 ms after the response and remained on the screen until the participants pressed the start key again. Errors were signaled by a tone.

There were five different shift-mode conditions, arranged in blocks as follows. The first condition comprised four different blocks (each of 64 trials) in which task as well as target level remained constant. In one of the blocks the odd-even task was required for the global target level, whereas in another block the same task had to be performed for the local level. There were two corresponding blocks for the less-greater task.

The second condition consisted of two blocks (each of 128 trials) in which the target level was fixed but the tasks were randomized. In one block the targets occurred always at the local level, and in the other they occurred always at the global level. The third condition consisted of two blocks (each of 128 trials) in which the task was constant but the target levels were randomized. In one block the odd-even task was always required, and in the other block the less-greater task was always required.

The fourth condition comprised only one block (of 256 trials) in which the tasks as well as the target levels were randomized independently. Finally the fifth condition consisted of two blocks (each of 128 trials) in which tasks and levels varied together. That is, in one block, the lessgreater task and the odd-even task were linked to the local level and the global target level, respectively. In the other block, the combination was reversed. This condition corresponded to the "shift both" condition in Experiment 1 of Allport et al. (1994).

The last 4 blocks were divided into experimental blocks of 64 trials each. Thus, altogether, there were 20 experimental blocks, which were distributed over two 1-hr main sessions. Each block type occurred once in each session, and the order of blocks within each session was randomized. There was also a preliminary training session, in which one block of 32 trials for each shift-mode type was run. Altogether, there were 1,280 experimental trials for each participant.

\section{Results}

\section{Response Times}

Only the latencies of correct responses were entered into the data analysis. To assess the effects of task and level shifts and their interaction, we examined mean latencies using analyses of variance (ANOVAs). Initially, we conducted a two way ANOVA with repeated measurements on the task mode (constant or randomized), and level mode (constant or randomized) variables the data for the condition involving the independent tasks and levels were used as task-randomized/levelrandomized data set.

The analysis revealed a significant main effect of task mode, $F(1,9)=20.16, p<.01, M S E=691.2$. Constant tasks led to faster judgments $(M=556 \mathrm{~ms})$ than randomized ones $(M=594$ ms). Also, the level-mode variable was significant, $F(1,9)=$ 41.71, $p<.001, M S E=575.6$. Responses under a constant target level were faster $(550 \mathrm{~ms})$ than responses under randomized levels $(599 \mathrm{~ms})$. Most important, the interaction between task mode and level mode was far from significance $(p=.37)$. The data are shown in Figure 3.

On the other hand, when we used the data for the condition with linked tasks and levels as task-randomized/level-randomized data set, the main effects remained significant, but the interaction between task mode and level mode was also significant, $F(1$, 9) $=7.62, p<.05, M S E=440.6$. When tasks and levels varied in a linked manner, the response times were similar to those in the slowest single-shift condition (i.e., where merely the target level varied; $588 \mathrm{~ms}$ vs. $583 \mathrm{~ms}$ ).

\section{Preparation Times}

Preparation times were analyzed in the same way as response times. First, we computed a two-way ANOVA with repeated measurements on the task mode (constant or randomized) and level mode (constant or randomized) variables. The data for the condition in which tasks and levels varied independently were 

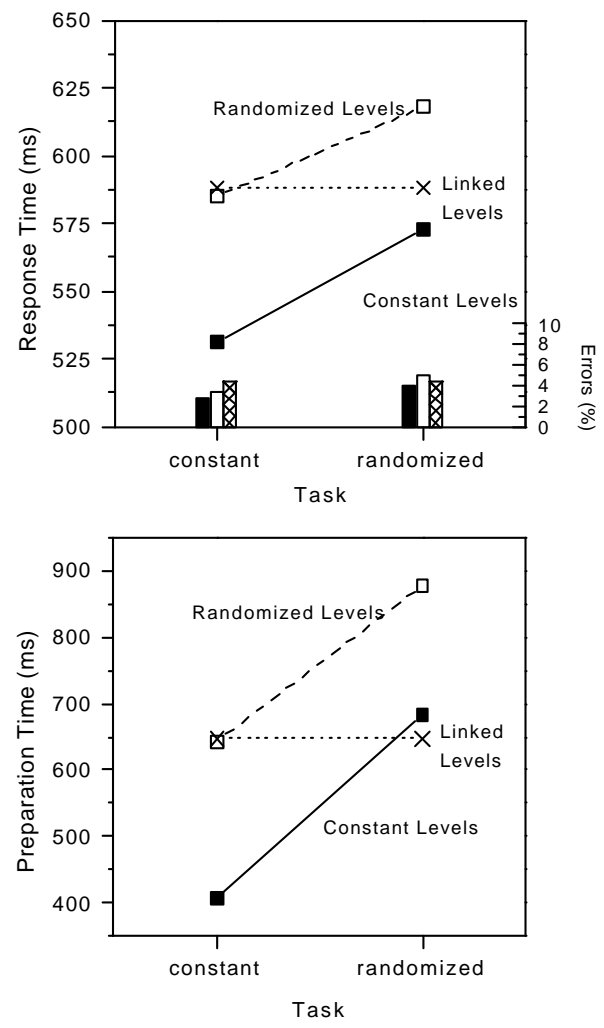

Figure 3. Results of Experiment 1. Top: Response times and errors for the different shift conditions. Bottom: Preparation times.

used as task-randomized/level-randomized data set. The analysis revealed a significant main effect of task mode, $F(1,9)=19.02$, $p<.01, M S E=35,541$. Mean preparation time for constant tasks was shorter $(M=525 \mathrm{~ms})$ than that for randomized tasks $(M=$ $781 \mathrm{~ms})$. Also, the level-mode variable was significant, $F(1$, 9) $=12.81, p<.01, M S E=36,274$. The participants spent less preparation time for constant levels $(545 \mathrm{~ms})$ than for randomized ones $(761 \mathrm{~ms})$. As was the case with response times, the interaction between the two variables was far from significance $(p=.418)$.

However, when we entered the data for the condition in which tasks and levels were linked into the analysis, the interaction between task mode and level mode was significant, $F(1,9)=8.23$, $p<.05, M S E=22,765$. In this case, the preparation times for the concurrent shifts were similar to those when only the target level was randomized (647 ms vs. $643 \mathrm{~ms}$ ). Preparation time data are also shown in Figure 3.

\section{Error Rates}

On average, errors occurred only on $4 \%$ of the trials. Because they pointed in the same direction as the response time data, they were not analyzed further.

\section{Discussion}

The results show that residual shift costs for linked tasks and dimensions were similar in amount to those in conditions in which only a single component varied. Thus, despite our different dimensions and other methodological differences, we replicated Allport et al.'s (1994) result in this respect. However, our data clearly show that independently varying tasks and levels produced more shift costs than linked components. Moreover, the costs for task shifting and for dimension shifting were additive. These results support our AC hypothesis. The selection of two independent components during task execution requires additional attentional control and, consequently, increases residual shift costs.

Interestingly, the time spent by the participants on preparation mirrored the pattern of their response times. Also, preparation time increased under shift conditions, and the effects of the individual shift conditions summed for independent task and dimension shifts. Thus, increasing preparation time did not prevent additional shift costs, which is in line with our hypothesis.

The question arises as how to the extra shift costs are distributed across trials in the independent condition. In this respect, it is important to note that our trial sequences were different from those of Allport et al. (1994). Because they used alternating tasks, their participants had to shift on every trial. In our experiment, the trials were randomized. Thus, in the linked condition, either no shift was required from one trial to the next or a concurrent shift was required. In conditions with independent components, there were trials with no shift, trials with a single-component shift, and trials with concurrent shifts. It is possible that, in this condition, task execution required more control only on trials on which two components had to be shifted. In this case, the concurrent-shift trials were responsible for the additional costs. However, it is also conceivable that overall performance was reduced in the independent condition. For instance, task execution could have proceeded in a highly controlled mode irrespective of whether a single component or a concurrent shift was required on a specific trial. The question of which of these possibilities holds was tested in the next experiment.

Thus far, our data are in line with the AC hypothesis. However, this does not imply that they could not be explained by alternative accounts. For instance, if we regard the number of activation patterns across the involved control units, then it is obvious that four patterns compete in the independent condition, as compared with two patterns in the linked condition. Therefore, the additional shift costs could be due to an increased amount of task cuing (cf. Rogers \& Monsell, 1995). Furthermore, because the number of control patterns corresponded to the number of involved pathways (see Figure 2), the number of competing pathways also increased in the independent condition. Thus, according to the proactiveinterference hypothesis (Allport et al., 1994), this might have increased the amount of cross talk and, consequently, shift costs. These considerations show that there are several potential sources that might have contributed to the additional residual shift costs in the independent condition. Whether there is a contribution of cross talk was examined in the next experiment.

\section{Experiment 2}

In Experiment 2, we investigated the contributions of concurrent shifts and of cross talk to the extra residual shifts for independently varying tasks and dimensions. To examine the effect of concurrent shifts, we included a condition in which tasks and target levels 
varied independently but no concurrent shifts occurred. If the extra costs observed in the previous experiment were due to concurrent shifts, then they should be reduced in this condition.

If cross talk is responsible, at least in part, for the extra costs, then there should be more cross talk in the independent condition than in the linked condition. To test this prediction, we examined consistency effects. As can be seen in Figure 2, there are different possibilities of how the pathways can interfere, each of which corresponds to a specific consistency type. Here we consider only two of these types: Task consistency and level consistency. ${ }^{1}$ Level consistency concerns response-compatibility relations between the levels of a stimulus. Specifically, a stimulus was regarded as level inconsistent if the numerals on the two levels were response incompatible with respect to the required task; otherwise, it was regarded as level consistent. On the other hand, task consistency concerns the response-compatibility relation between the tasks for a given level. Accordingly, a stimulus was defined as task inconsistent if the numeral at the relevant level was response incompatible with respect to the two tasks; otherwise, it was defined as task consistent.

The different consistency types can be understood by inspecting Figure 2. A specific example is indicated by the different arrow types. Assume that the task is a less-greater judgment with the numeral at the local level (8). The appropriate pathway is indicated by the large arrows. In this example, the stimulus is level inconsistent, because the numerals on both levels map to different responses ( 3 is less, and 8 is greater). The competing pathway is indicated by the dashed arrows. On the other hand, the stimulus is task consistent, because both judgments with the numeral at the relevant level ( 8 is even and greater) map to the same response. The respective facilitatory activation flow is represented by the stippled arrows. We consider the difference between the performance for consistent stimuli and the performance for inconsistent stimuli as a measure of cross talk. It bears mention that, in Allport et al. (1994), all stimuli were inconsistent.

A closer look at the number of shift trials in the different blocks reveals a confounding in Experiment 1 that needs to be addressed. In a block of trials for the linked condition, we expect a shift on about $50 \%$ of the trials. This is the same for blocks with singlecomponent shifts. However, when both components vary independently, we expect a shift on about $75 \%$ of the trials: pure dimension shifts on $25 \%$ of the trials, pure task shifts on $25 \%$ of the trials, and concurrent shifts on $25 \%$ of the trials. At least for level shifts, Lamb, London, Pond, and Whitt (1998) have shown that the frequency also of shifting can be an important variable.Thus, because shift frequency also could have increased the shift costs in the independent condition, this issue was addressed in the next experiment.

Altogether, four variables were manipulated in Experiment 2. One variable concerned the shift mode: One condition was identical to the randomized mode in the previous experiment, a second condition was identical to the linked mode, and a third condition was identical to the first one, except that no concurrent shifts occurred. The second factor concerned the number of shifts within a block of trials. There were two shift frequencies: $50 \%$ and $75 \%$. The third and fourth variables concerned task consistency and level consistency, respectively.

\section{Method}

Eight participants (2 male and 6 female), who ranged in age from 19 to 38 years, took part in the experiment. All were right-handed (by self-report) and had normal or corrected-to-normal vision. The apparatus and the procedure were the same as in the previous experiment.

Two types of consistency were considered: level consistency and task consistency. With respect to level consistency, a stimulus was considered consistent if, for the relevant task, the numerals at both levels belonged to the same response category; otherwise, it was defined as inconsistent. Concerning task consistency, a stimulus was regarded as consistent if both tasks led to the same response for the numeral at the relevant level; otherwise, it was considered inconsistent.

The trial sequences were produced in advance and edited such that the different requirements were met. Different exemplars for each condition were constructed and assigned randomly to the participants. Both combinations of task and level were realized for the linked condition. Twelve blocks of 80 trials each were distributed over two 50-min sessions. Altogether, there were 40 trials for each of the 24 conditions. The order of experimental blocks was random for each participant, except that a block could not be repeated. A preliminary training session involved approximately seven blocks of 40 trials each.

\section{Results}

\section{Response times}

The latencies of correct responses were entered into a four-way ANOVA with repeated measurements on shift mode (randomized, linked, or randomized without concurrent shifts), shift frequency (50\% or $75 \%$ ), task consistency (consistent or inconsistent), and level consistency (consistent or inconsistent). The analysis revealed a significant main effect of shift mode, $F(2,14)=12.01$, $p<.001, M S E=340.0$. Responses in the linked condition were faster $(M=575 \mathrm{~ms})$ than those in the other two conditions $(M=$ $589 \mathrm{~ms}$ for randomized and $M=587 \mathrm{~ms}$ for randomized without concurrent shifts). Shift frequency had no significant effect ( $p=$ .695). The data are shown in Figure 4.

Also the task-consistency and level-consistency variables were significant, $F(1,7)=48.24, p<.001, M S E=4,736$, and $F(1$, 7) $=24.46, p<.01, M S E=459.8$, respectively. However, there was no reliable interaction between consistency and task mode. The task-consistency effects were $67 \mathrm{~ms}$ for the linked condition, $71 \mathrm{~ms}$ for the randomized condition, and $69 \mathrm{~ms}$ for the condition without concurrent shifts. The corresponding level consistency effects were $13 \mathrm{~ms}, 10 \mathrm{~ms}$, and $24 \mathrm{~ms}$. The effects of both consistency types were almost perfectly additive: $79 \mathrm{~ms}$ in the linked condition, $81 \mathrm{~ms}$ in the randomized condition, and $93 \mathrm{~ms}$ in the condition without double shifts.

\section{Preparation Times}

An ANOVA for the first two variables was also computed for preparation times. This analysis revealed a significant main effect of shift mode, $F(2,14)=5.76, p<.05, M S E=33,280$. The participants spent less time on preparation in the linked condition

\footnotetext{
${ }^{1}$ Actually, there is also level-task consistency. This type concerns the response tendency triggered by the numeral at the irrelevant level with respect to the irrelevant task. However, for simplicity, and because we expected small effects, we did not examine this type of interference.
} 


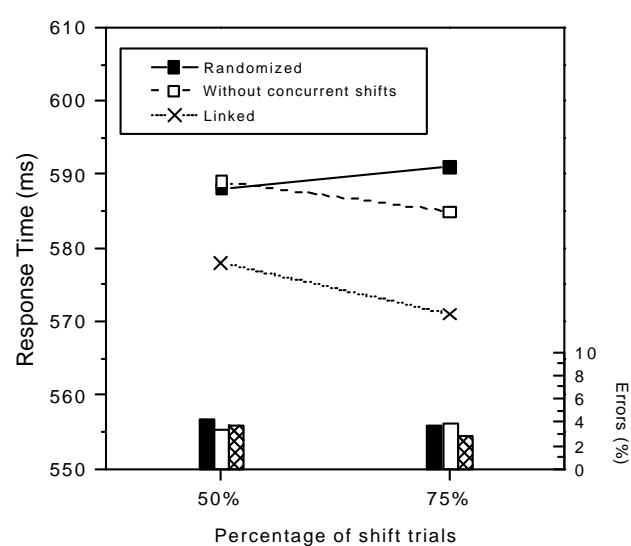

Figure 4. Results of Experiment 2.

$(M=815 \mathrm{~ms})$ than in the other two conditions $(M=924 \mathrm{~ms}$ for randomized, and $M=880 \mathrm{~ms}$ for randomized without concurrent shifts). Frequency had no reliable effect $(p=.729)$.

\section{Error Rates}

On average, the error rate was $3.62 \%$. Because there was no indication of a speed-accuracy trade-off (see Figure 4), error rates were not analyzed further.

\section{Discussion}

First of all, shift frequency had no effect and therefore cannot explain the results of Experiment 1. This is at odds with the results of Lamb et al. (1998), who did find effects of shift frequency. However, they considered frequencies of $0 \%, 50 \%$, and $100 \%$, which covered a range considerably larger than that in our experiment. Moreover, they examined only shifts between levels. These differences could be responsible for the discrepant results.

Concerning the additional shift costs for independent components relative to linked ones, our results demonstrate that concurrent shifts as such are not responsible. The costs remained similar, even when no concurrent shifts were required in a block of trials. This indicates that overall performance was reduced when tasks and levels varied independently. That this reduction was not due to an increased amount of cross talk between the involved pathways is obvious by the fact that the consistency effects were similar for independent and linked components.

Together, these results are in line with the AC hypothesis. They indicate that task execution generally required more attentional control in the independent condition. This suggests that the participants worked through an obligatory two-step attentional selection process on each trial. This would also explain why frequency had no effect. Moreover, because concurrent shifts did not contribute to the extra shift costs, it follows that the sequential effects should be similar for concurrent and single-component shifts. To test this prediction, we examined sequential effects in the next experiment. This also offered the opportunity to test Rogers and Monsell's (1995) hypothesis that sequential shift costs occur only on the first trial of a run.
That cross talk did not vary with shift costs is surprising, because we know that both effects can covary considerably. For instance, cross talk usually increases in conditions with randomized components relative to constant ones (e.g. Hübner, 1997, 2000). To examine whether this also held for the present case, we included constant conditions in the next experiment and compared the corresponding consistency effects and shift costs with those in randomized conditions.

To examine sequential effects reliably in the different conditions, we had to collect a large amount of data. Therefore, to keep the experimental effort within our limits, we did not include a condition with linked components. However, our data allowed us to examine responses to individual tasks and levels separately. Particularly interesting in this respect are dominance relations. With respect to levels, responses to the global level are usually faster than those to the local level, and the interference from global to local is larger than vice versa (cf. Hübner, 1997; Navon, 1977). Similarly, less-greater judgments are usually faster than odd-even judgments, and there is a corresponding interference asymmetry (e.g., Allport et. al. 1994; Sudevan \& Taylor, 1987). How does dominance affect shift costs? Allport et al. found in one of their experiments that shifts from a difficult to an easy task produced more costs than vice versa. They explained this counterintuitive result by assuming that a nondominant $\mathrm{S}-\mathrm{R}$ mapping requires a strongly imposed task set so that active disengagement is necessary for shifting to a dominant task. To investigate this issue, we considered dominance relations in the next experiment. According to our AC hypothesis, more control effort should be required for executing a nondominant task against the competition of a dominant one than vice versa.

\section{Experiment 3}

\section{Method}

Twelve participants ( 6 male and 6 female), who ranged in age from 23 to 39 years, took part in the experiment. All were right-handed (by self-report) and had normal or corrected-to-normal vision. The apparatus and the procedure were the same as in Experiment 1. However, additional variables were introduced. In all, six experimental variables were considered: task (odd-even or less-greater), level (local or global), task mode (constant or randomized), level mode (constant or randomized), task consistency (consistent or inconsistent), and level consistency (consistent or inconsistent). Together, there were 64 different conditions, arranged in the same manner as the first four block types in Experiment 1. Here, however, the number of trials was doubled. Accordingly, there were 128 trials in each of the 16 experimental blocks, distributed over four 1-hr main sessions. Each block type occurred once in each session, and the order of blocks within each session was randomized. In addition, there was a preliminary training session in which 9 different blocks of 64 trials were run. Altogether, there were 32 experimental trials for each condition and participant (i.e., 2,048 trials for each participant).

\section{Results}

\section{Response Times}

The latencies of correct responses were subjected to a six-way ANOVA with repeated measurements on all variables: task (odd or even), level (local or global), task mode (constant or randomized), 
level mode (constant or randomized), task consistency (consistent or inconsistent), and level consistency (consistent or inconsistent).

Task mode and level mode. We first present the main results concerning the task-mode and level-mode variables. The analysis revealed a reliable effect of task mode, $F(1,11)=14.63, p<.01$, $M S E=73,880$. Faster responses occurred with a constant task than with variable ones $(M=594 \mathrm{~ms}$ vs. $670 \mathrm{~ms})$. A similar result was obtained for level mode (587 ms vs. $677 \mathrm{~ms}), F(1,11)=25.3$, $p<.001, M S E=60,511$. As can be seen in the upper panel of Figure 5, there was no interaction between these two variables.

Target level and level consistency. The analysis also revealed a significant main effect of target level, $F(1,11)=9.10, p<.05$, MSE $=21,813$. As expected, responses to the global target level were faster $(\mathrm{M}=616 \mathrm{~ms})$ than those to the local level $(M=648$ $\mathrm{ms})$. Also, the levelconsistency factor was significant $F(1$, $11)=21.59, p<.001, M S E=1,327$. Latencies for consistent stimuli were shorter $(626 \mathrm{~ms})$ than those for inconsistent stimuli (638 ms). However, there was a significant interaction between level and level consistency, $F(1,11)=7.22, p<.05$, $M S E=1,019$. As can be seen in the left panel of Figure 6 the difference between the latencies for consistent and inconsistent trials was smaller for the global level $(6 \mathrm{~ms})$ than for the local one (18 ms).

Task and task consistency. The main effect of task was significant, $F(1,11)=15.41, p<.01, M S E=10,971$. Less-greater judgments were faster $(M=617 \mathrm{~ms})$ than odd-even ones $(M=$ $647 \mathrm{~ms}$ ). Also task consistency had a reliable effect (599 ms vs. $665 \mathrm{~ms}), F(1,11)=97.42, p<.001, M S E=8,491$. However, there was a significant interaction between task consistency and task, $F(1,11)=11.37, p<.01, M S E=3,769$. As shown in the right panel of Figure 6 , the effect of task consistency was smaller for the less-greater task $(50 \mathrm{~ms})$ than for the odd-even task (80 ms).

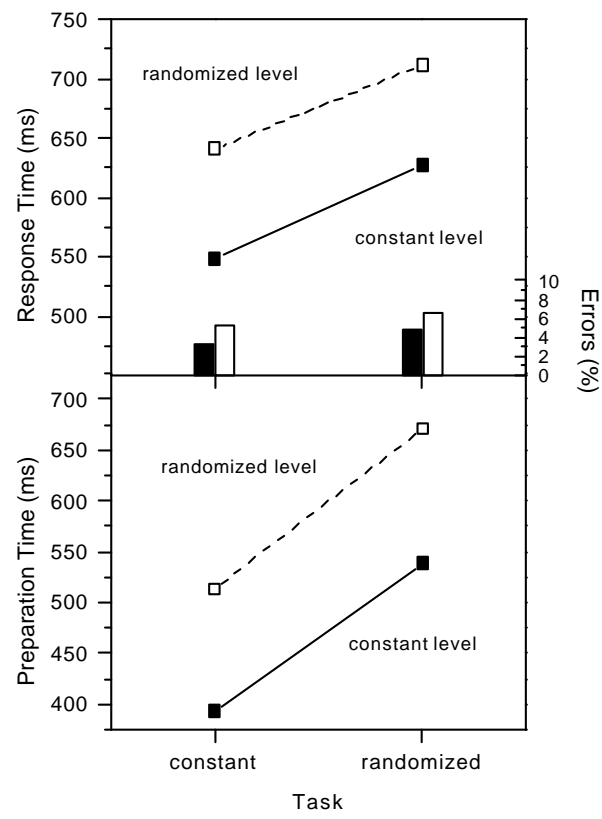

Figure 5. The effects of task mode and levelmode in Experiment 3. Top: Response times and errors. Bottom: Preparation times

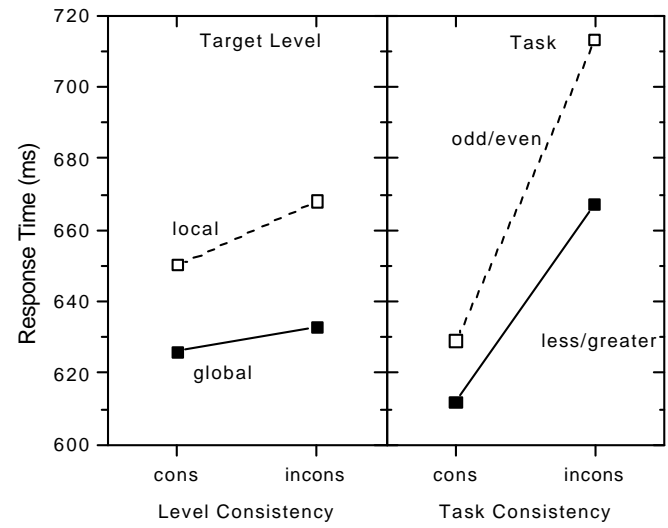

Figure 6. Results of Experiment 3. Left: Interaction between target level and level consistency. Right: Interaction between task and task consis tency. cons $=$ consistent; incons $=$ inconsistent.

Task consistency also interacted with task mode, $F(1$, 11) $=20.86, p<.001, M S E=2,791$. The task-consistency effect was smaller in constant-task conditions (49 ms) than in conditions with randomized tasks ( $89 \mathrm{~ms}$ ). In addition, task consistency was affected by le vel mode, $F(1,11)=16.62, p<.01, M S E=237.5$. Task consistency produced a slightly smaller effect in conditions with a constant target level $(61 \mathrm{~ms})$ than in conditions with a randomized target level (70 ms).

However, there was also a reliable three-way interaction among task consistency, target level, and level mode, $F(1,11)=5.38$, $p<.05, M S E=1,820$. Although level mode did not affect task consistency for numerals at the global level $(67 \mathrm{~ms}$ vs. $62 \mathrm{~ms})$, task consistency was smaller under a constant level than under randomized levels for numerals at the local level $(55 \mathrm{~ms}$ vs. $79 \mathrm{~ms})$. The interaction between task consistency and level mode also depended on the task, $F(1,11)=6.37, p<.05, M S E=290.4$. For less-greater judgments, the task-consistency effect was similar irrespective of level mode ( $49 \mathrm{~ms}$ vs. $53 \mathrm{~ms}$ ). However, for odd-even judgments, task consistency was increased under randomized levels ( $73 \mathrm{~ms}$ vs. $88 \mathrm{~ms}$ ). Finally, it should be noted that despite the different interactions in which task consistency and level consistency were involved, they did not interact with each other. That is, their effects were additive.

\section{Preparation Times}

The preparation times for trials with correct responses were entered into a four-way ANOVA with repeated measures on all variables: task mode (constant or randomized), level mode (constant or randomized), task (odd-even or less-greater), and level (global or local). Level mode $(M=466 \mathrm{~ms}$ vs. $592 \mathrm{~ms} ; F(1$, 11) $=22.96, p<.001, M S E=132,198)$, as well as task mode $(M=453 \mathrm{~ms}$ vs. $605 \mathrm{~ms} ; F(1,11)=11.1, p<.01, M S E=$ $399,485)$, had a significant effect. As can be seen in the lower panel of Figure 5, the pattern was similar to that of the response times.

\section{Sequential Effects}

Variable tasks and levels. First, repetition effects were analyzed for the condition in which the tasks as well as the levels 
varied. A four-way ANOVA was computed with the following variables: task (odd-even or less-greater), level (global or local), task repetition (repetition or nonrepetition), and level repetition (repetition or nonrepetition). Only the main effects of the last two variables, and their interactions with the other variables are of interest.

Task repetitions ( $M=778 \mathrm{~ms}$ vs. $797 \mathrm{~ms}), F(1,11)=7.76, p<$ $.05, M S E=2,080$, as well as level repetitions $(M=780 \mathrm{~ms}$ vs. $796 \mathrm{~ms}), F(1,11)=27.10, p<.001, M S E=455.7$, produced significant benefits. However, there was an interaction between the two repetition types, $F(1,11)=6.98, p<.05, M S E=458.6$. There was a benefit only when both the level and the task repeated. In other words, and as a post hoc comparison revealed, there were no extra costs for concurrent shifts relative to the most costly single-component shifts, $t(47)=1.26, p=.21$.

Furthermore, the interaction between level repetition and level was reliable, $F(1,11)=8.05, p<.05, M S E=2,755$. The repetition benefits were larger for the local level $(38 \mathrm{~ms})$ than for the global level $(6 \mathrm{~ms})$. Moreover, there was an interaction between level repetition and task, $F(1,11)=11.52, p<.01, M S E=$ 360.1. The level-repetition benefits were larger for odd-even judgments $(25 \mathrm{~ms})$ than for less-greater judgments $(7 \mathrm{~ms})$.

Variable levels. A repeated measures three-way ANOVA was computed with the following variables: task (odd-even or lessgreater), level (global or local), and level repetition (repetition or nonrepetition). In the present analysis, only level repetition and its interactions with the other two variables are of interest.

First of all, the repetition variable was significant, $F(1$, $11)=13.92, p<.01, M S E=3,828$. Responses on repetition trials were faster $(M=662 \mathrm{~ms})$ than those on nonrepetition trials $(M=$ $709 \mathrm{~ms})$. However, there was an interaction between level repetition and target level, $F(1,11)=5.07, p<.05, M S E=828.1$. The repetition benefit was larger for the local level $(60 \mathrm{~ms})$ than for the global one $(34 \mathrm{~ms})$. In addition, there was an interaction between level repetition and task, $F(1,11)=15.92, p<.01, M S E=969.9$. As can be seen in Figure 7, the level repetition benefit was larger in blocks with odd-even judgments $(73 \mathrm{~ms})$ than in blocks with less-greater judgments (22 ms).

Variable tasks. Next, we analyzed the conditions in which the target level remained constant but the task varied. A three-way ANOVA was computed with the variables task (odd-even or less-greater), level (global or local), and task repetition (repetition or nonrepetition). Here, merely the effect of repetition and its interactions with the other variables are of interest.

The repetition variable was significant, $F(1,11)=23.18, p<$ $.001, M S E=2,486$. Responses on repetition trials were faster $(M=655 \mathrm{~ms})$ than those on nonrepetition trials $(M=704 \mathrm{~ms})$ However, repetition interacted with task, $F(1,11)=9.89, p<.01$, $M S E=591.8$. Repetition benefits were larger for odd-even judgments $(65 \mathrm{~ms})$ than for less-greater judgments $(34 \mathrm{~ms})$. The difference can be seen in Figure 7.

\section{Run Length of Three}

For testing the hypothesis that sequential shift costs occur only on shift trials (Rogers \& Monsell, 1995), runs of length three were analyzed for conditions with single-component shifts. We tested whether there was a decrease in response time between Position 2 and Position 3 of a run. It turned out that there was a significant

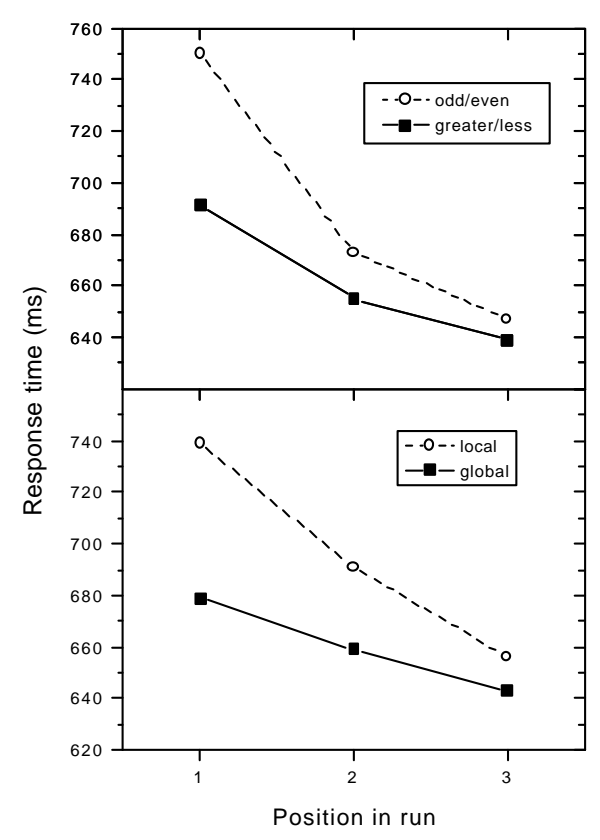

Figure 7. Repetition effects in Experiment 3 for runs of length three in conditions with single shifts. Top: Results for task repetitions. Bottom: Results for level repetitions.

decrease for task, $t(23)=2.79, p<.05$, as well as for dimension, $t(23)=3.55, p<.01$. Data are shown in Figure 7 .

\section{Error Rates}

Errors occurred, on average, in $5.04 \%$ of the trials. They largely showed the same pattern as the latencies, as can be seen in Figure 5. Because there was no indication of a speed-accuracy trade-off, errors were not analyzed further.

\section{Discussion}

Shift costs were generally larger in Experiment 3 than in Experiment 1 . Nevertheless, the main pattern of results was similar. Also, here the costs for variable levels and variable tasks were additive. This shows that costs might vary depending on the circumstances or the specific sample of participants but that their proportions remain largely unaffected.

As expected, the sequential effects were similar for concurrent and for single-component shift trials. In other words, there was a benefit only for a repetition of both components from one trial to the next. Any change produced a similar amount of costs, irrespective of whether one or both components differed. This is compatible with our results in Experiment 2, in which it did not matter in regard to overall costs whether there were concurrent shifts. Moreover, our results demonstrate that the residual shift costs are not due to sequential effects. In conditions with one variable component, the repetition effects were considerably larger than in conditions with two variable components, although the shift costs were smaller in the former case. Thus, the sequential effects were smallest in conditions with the largest overall costs. 
Concerning dominance, the benefit was larger for the repetition of a nondominant task or level than for the repetition of a dominant component. Furthermore, and different from the results of Rogers and Monsell (1995), performance improved as well for the second repetition in a run, although to a smaller extent than for the first one. Thus, our results do not support their stimulus-cued completion hypothesis.

In the previous experiments we found that cross talk did not contribute to the increase in shift costs from the linked to the independent condition. Therefore, we examined in the present experiment whether this also holds for costs relative to the constant conditions. It turned out that, in this case, cross talk increased but only with respect to task consistency. That is, task-consistency effects increased under randomized tasks as well as under randomized levels, whereas level consistency remained unaffected. Because negative effects of cross talk are usually larger than facilitatory effects (e.g., Rogers \& Monsell, 1995), this indicates that cross talk contributed to the shift costs. However, it is again obvious that the shift costs cannot be explained by cross talk alone. If we compare the latencies for consistent stimuli in the constant conditions with those in conditions in which both components varied, the difference is about $130 \mathrm{~ms}$. The corresponding difference for inconsistent stimuli is $190 \mathrm{~ms}$. Thus, although cross talk contributed considerably to the shift costs, a large proportion remained unexplained.

We have already considered dominance effects with respect to sequential costs. In a similar manner we can ask how dominance affected overall shift costs. As mentioned, shift costs were often unaffected by dominance relations (e.g. Hübner, 1997; Hübner \& Backer, 1999; Sudevan \& Taylor, 1987) or affected in a contraintuitive way (cf. Allport et al., 1994, Experiment 4). In the present experiment, the effects were in the intuitively expected direction. That is, randomization produced more costs for the nondominant tasks and levels than for the dominant components.

Taken together, the present results also support the AC hypothesis. Cross talk and repetition effects account only for a small fraction of shift costs. Thus, the larger part seems to be due to the control effort required during task execution. An important characteristic of the AC hypothesis is that task execution is considered as an active process that involves attentional control, whereas other accounts assume that voluntary control takes place only during task preparation. According to these alternative hypotheses, execution proceeds in a passive way; a reduction in speed is due to processing delays caused by interference, either between S-R mappings or between control schemas. Here we could show that at least the competition between S-R mappings (cf. Allport et al., 1994) is not sufficient for explaining residual shift costs.

Unfortunately, the issue is more difficult with respect to competing control schemas. If one regards the activation pattern of the control units (see Figure 2) for a specific combination of tasks and levels as a control schema, then it could be argued that our results are also compatible with task cuing (cf. Rogers \& Monsell, 1995). Under the assumption that the number of competing schemas determines the amount of shift costs, independently varying tasks and dimensions should produce more costs than linked ones. This follows, because four schemas are involved in the former case as compared with two in the latter one. Moreover, if one assumes that the interference between all of the relevant control schemas is effective on each trial, task cuing can even explain that concurrent shifts are not important for the overall costs in the independent condition.

Thus, up to now, many of our results can also be accounted for by the task-cuing hypothesis. What seems difficult to explain by task cuing is the additivity of shift costs. However, one could argue that there is possibly a nonlinear relationship between the number of involved control schemas and shift costs, that might explain our results. Thus, an experiment is required that convincingly demonstrates that not the number of involved schemas, but the number of controlled task components determines shift costs.

\section{Experiment 4}

The next experiment was conducted to show that task cuing is not sufficient for explaining residual shift costs. To attain this goal, we wanted to vary the number of involved control schemas without affecting the number of task components that require attentional control. If in this case, the amount of residual shift costs remained constant, this would definitely contradict task cuing and support the AC hypothesis. Our solution was to consider shift costs in conditions with three relevant control schemas. Task cuing predicts that shift costs should be smaller in this case than in the independent condition, in which four schemas are relevant. On the other hand, shift costs should be larger than in conditions with only two relevant schemas.

In conditions in which three control schemas are relevant, le vels and tasks are partially linked. That is, both tasks occur at one level, but only one task occurs at the other level. For instance, odd-even as well as less-greater judgments are required for the numerals at the global level, whereas exclusively odd-even judgments must be performed with the numerals at the local level. However, because tasks and levels are not uniquely linked, separate and controlled level selection and task selection should also be required in this case. Thus, the AC hypothesis predicts no difference in shift costs between conditions with partially linked and independent components.

\section{Method}

Eight participants ( 2 male and 6 female), who ranged in age from 18 to 27 years, took part in the experiment. All had normal or corrected-tonormal vision. The apparatus and the procedure were the same as in the previous experiments.

There were five different shift-mode conditions. In one condition only the levels varied, and in another only the tasks varied. Furthermore, there were blocks of trials in which tasks and levels varied independently and blocks in which they were perfectly linked. In addition, there was a critical condition with partially linked tasks and levels. That is, although both tasks occurred for the numerals on one level, only one task had to be performed with the numeral on the other level. Constant conditions occurred only during the training sessions.

Each of the first four conditions comprised 4 blocks of 64 trials each. The critical condition consisted of 4 blocks with 66 trials. The blocks of the latter condition corresponded to the four possible mixtures of three of four task-dimension combinations. In each of these blocks, each of the three task-dimension combinations occurred in one third of the trials. Altogether, there were 20 experimental blocks, distributed over two 1-hr main sessions. Each block type occurred twice in each session, and the order of blocks was balanced across participants to the extent possible. To control further for possible learning effects, we treated the temporal position of the blocks as a variable. At the beginning of the experiment, there was a 1-hr 
training session, in which constant as well as shift conditions occurred. Altogether, there were 1,288 experimental trials for each participant.

\section{Results}

The mean latencies of the correct responses were entered into an ANOVA with shift mode (variable task, variable level, linked components, partially linked components or independent components) and block position (1, 2, 3 or 4$)$ as variables. The shiftmode variable, $F(3,21)=9.10, p<.001, M S E=706.3$, and the position variable, $F(3,21)=16.62, p<.001, M S E=1,832$ were significant, whereas their interaction was not reliable $(p=.175)$. To test our hypotheses, we calculated planned comparisons. They revealed that the responses to stimuli in the partial condition were significantly slower than those in the linked condition, $t(31)=$ $2.29, p<.05$. However, they did not differ from responses in the independent condition $(p=.80)$. On the other hand, the mean latencies in the linked condition were not significantly different from those in the variable-task condition $(p=.31)$. The mean latencies for the different shift mode conditions can be seen in the upper panel of Figure 8.

Preparation times are shown in the lower panel of Figure 8. Planned comparisons revealed that the mean latencies for the linked condition were significantly slower than those for the partial condition, $t(31)=3.15, p<.01$. However, unlike the case with response time, the latency difference between the partial condition and the independent condition was significant as well, $t(31)$ $=2.21, p<.05$.
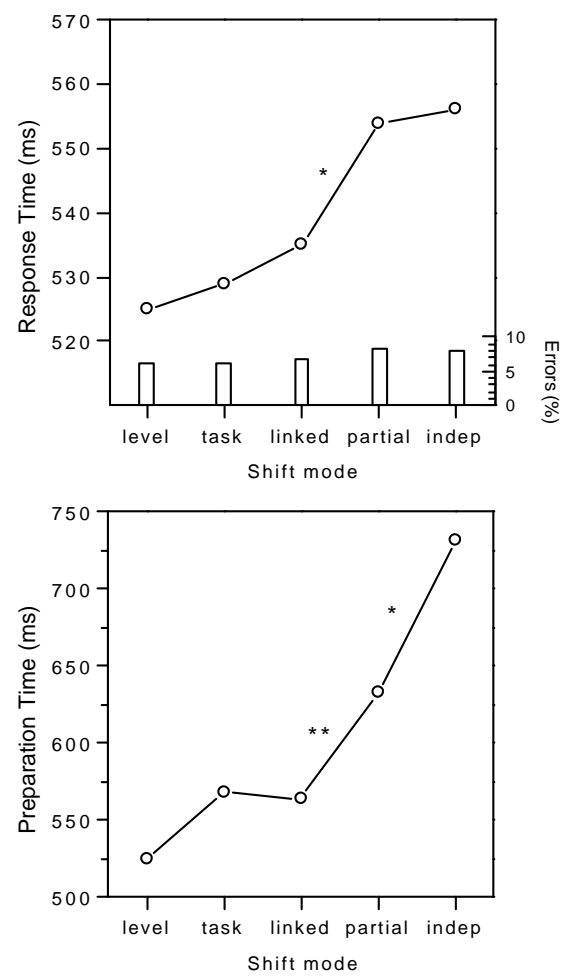

Figure 8. Results of Experiment 4. Top: Response times. Bottom: Preparation times. Asterisks denote significant differences between means. indep $=$ independent.
Errors occurred, on average, in $7.21 \%$ of the trials. They showed a similar pattern to the latencies, as can be seen in Figure 8 . Because there was no indication of a speed-accuracy trade-off, the errors were not analyzed further.

\section{Discussion}

Our results demonstrate that the number of involved control schemas did not determine the amount of residual shift costs. Although only three control schemas were relevant in conditions with partially linked levels and tasks, the shift costs were not reliably smaller than in the independent condition with four relevant schemas. This result is incompatible with the task-cuing account but strongly supports our AC hypothesis. It also indicates that partially linked levels and tasks indeed require selecting and processing each component separately. Each step had to be performed in a controlled mode and, therefore, consumed attentional resources. Thus, our results demonstrate again that the control effort spent for task execution determines shift costs.

On the other hand, preparation time varied systematically with the number of control schemas. This shows that, different from execution, the time spent for preparation increased with the set size of the relevant schemas rather than with the number of required selection steps.

\section{General Discussion}

The aim of the present experiments was to investigate the mechanisms that control and coordinate the execution of mental tasks under task-shift conditions. Because shifts between tasks usually produce costs in response time and errors, the question arises as to which specific mechanisms are responsible for this effect. Obviously, before a certain task can be performed, the mental system must be configured appropriately. It seems that parts of the configuration can be done in advance, and it is widely assumed that this is accomplished through some kind of unitary supervisory system. However, as much evidence shows, preliminary reconfiguration reduces shift costs but cannot eliminate them. That is, even after extensive preparation, residual costs remain. Curiously, these costs have not been attributed to supervisory control (e.g., Allport et al., 1994; Meiran, 1996; Rogers \& Monsell, 1995). Rather, it has been argued that they merely reflect passive mechanisms such as interference between competing S-R mappings or task schemas. Moreover, exogenous control processes are considered sufficient for finally eliminating the residual costs. For instance, Rogers and Monsell proposed in their twocomponent model that external stimulation is necessary and sufficient for completing the reconfiguration of the mental system.

Here we questioned this view and proposed an alternative explanation for residual shift costs. We hypothesized that, under shift conditions, supervisory or attentional control is also active during task execution. It was assumed that this is necessary for controlling the selection of appropriate task components, especially when there is competition from other processes. We reasoned, similar to Allport et al. (1994), that when the AC hypothesis holds, the control of two task components should produce more costs than the control of only one. To test this prediction, we examined performance in conditions in which shifts between stimulus levels (dimensions) as well as between tasks were required. 
To consider the mental structures possibly involved in performing our two-component task in some detail, we devised a theoretical framework (see Figure 2). It was assumed that execution proceeds at least through two selection and processing steps. First, the numeral must be selected from the prespecified level. Second, the required judgment has to be chosen and performed. The selections are controlled by control nodes that modulate the activation of the appropriate level and task nodes (cf. Cohen et al., 1990). According to the AC hypothesis, when the relevant level or task changes across trials, selections must be carefully controlled to guarantee that the correct judgment is made with the numeral at the right level. Consequently, the more components vary, the more control is required and the slower are responses.

Allport et al. (1994), who have compared such conditions, observed the same amount of costs for single-component shifts and concurrent shifts between stimulus dimensions and tasks. Therefore, they concluded that attentional control plays no role in shift costs. However, their results are rather specific, because they considered only shifts between uniquely linked components. In our experiments, we were able to show that the control of two task components indeed produces more costs than the control of only one, provided that the components are independent. This supports our AC hypothesis. Moreover, the shift costs for two independent components were even similar to the sum of the costs for the individual components.

On the other hand, we found, as did Allport et al. (1994), that controlling two linked components produced similar costs to controlling a single component. Within our framework, this can be explained by assuming that, in such conditions, the control nodes for level selection are strongly associated with the corresponding nodes for task selection. Consequently, task selection occurs automatically with level selection and therefore needs no extra attentional control.

These results and considerations raised the question of whether the increased shift costs in conditions with independent components were merely due to an increased control effort on trials on which both components changed. As the results of Experiment 2 revealed, this was not the case. Rather, the processing of independently varying task components reduced overall performance, even that on non shift trials. This result was further supported by the sequential effects examined in Experiment 3.

At this points we could demonstrate that our results strongly support the AC hypothesis. The next step was to show that they cannot be explained by alternative accounts that attribute residual shift costs to passive mechanisms such as interference. For instance, Allport et al. (1994) proposed that shift costs reflect proactive interference between competing S-R mappings. In our framework, S-R mappings correspond to the horizontal pathways from the stimulus to the responses. The interference between these pathways was denoted as cross talk (cf. Rogers \& Monsell, 1995) and measured by consistency effects. According to Allport et al., the number and strength (dominance) of the competing pathways should determine the amount of cross talk and, consequently, the amount of shift costs. However, in Experiment 2, consistency effects were similar for independent and linked components, at though they produced different shift costs. Furthermore, even though in Experiment 3 consistency effects increased under shift conditions relative to constant conditions, and dominance had an effect as well, cross talk could not account for the observed costs.
For instance, variable levels produced considerably smaller consistency effects than variable tasks. Nevertheless, both variations produced about the same amount of shift costs. This demonstrates that cross talk explains (if at all), only a small fraction of the shift costs.

A further possible account for residual shift costs was task cuing, that is, the interference between competing control schemas (cf. Rogers \& Monsell, 1995). Similar to the case for pathways, one would expect that an increasing number of involved schemas should increase interference and, consequently, shift costs. On the other hand, according to the AC hypothesis, it is not the number of control schemas that is relevant for shift costs, but the number of attentionally controlled selection or processing steps. To examine which account holds, we used a condition with three relevant control schemas. In this case, either task could occur for the numeral at one level, but only one task was relevant for the numeral at the other level. Although levels and tasks were partially linked under this condition, we expected that two controlled selection steps were necessary on each trial. Thus, according to the AC hypothesis, control effort and, consequently, shift costs should not be substantially reduced relative to the independent condition, in which four control schemas are relevant. This prediction was tested in Experiment 4, which showed that three relevant control schemas produced more costs than two schemas but an amount similar to four schemas. This result contradicts task cuing and strongly supports our AC hypothesis.

Thus, together our results indicate that neither the number of competing pathways nor the number of competing control schemas determines the amount of residual shift costs. What matters is the number of variable task components that need attentional control during execution. This shows that residual shift costs are not merely due to passive mechanisms such as cross talk and task cuing and therefore uninteresting for investigating supervisory control. Rather, they are the result of active attentional processes that control and coordinate the processing steps during task execution. However, task cuing and cross talk are not without effect. On the one hand, they impose a controlled task execution. The participants seem to adopt a certain processing strategy throughout a block of trials that ensures successful task execution. On the other hand, cross talk or consistency affects the amount of required control on individual trials.

Our account also explains why the sequential effects were relatively small in the independent condition. Under these circumstances, level selection and task selection were performed in a highly controlled mode. Therefore, it mattered little whether one or both components varied from one trial to the next. Merely when both components remained constant, there was a small repetition benefit. On the other hand, the sequential effects were larger in conditions with one variable component. Moreover, nondominant components profited more from repetitions than dominant ones. Because less attentional control was required in these conditions, it is conceivable that some of the spared resources were used for increased engagement on a trial-by-trial basis. Interestingly, repetition benefits increased with run length. This shows that there was no fixed optimal configuration state that could be reached immediately after the first shift trial, as proposed by the stimuluscued completion hypothesis (Rogers \& Monsell, 1995).

Because we were interested in residual shift costs, our participants could prepare the task on each trial as long as they wanted. 
Out of curiosity, we also registered and examined the duration of this interval. In the first three experiments the pattern of the preparation times was analogous to that of the response times. However, in the last experiment the patterns differed. The time spent for preparation increased systematically with the number of involved control schemas. This suggests that the participants prepared a task by choosing the appropriate control schema from the set of relevant ones. Such an interpretation is similar to that of Dixon (1981) and Dixon and Just (1986), who assumed that task preparation consists of loading a mental program that controls the distribution of attentional resources during task execution. Although such an account is perfectly compatible with our AC hypothesis, it is rather speculative. Actually, we do not know what exactly happens during the preparation interval, or what it means to reconfigure the mental system.

One might ask whether our account holds generally or only for our two-component tasks. As Monsell (1996) stated, a task can be considered as consisting of one or multiple components, depending, for example, on viewpoint. From our perspective, what matters is whether a decision or selection step is required during task execution. Because this is the case as well for shifts between tasks with one component, we believe that our conclusions hold generally.

Finally, it should be noted that whereas our AC hypothesis has specifically been developed to explain residual shift costs in task shifting, there are similar ideas in other areas. As already mentioned, Norman and Shallice (1986), in their SAS model also emphasized the importance of supervisory control for goat directed task execution. It is assumed that such a mechanism allows individuals to apply control strategies in a flexible manner according to the task requirements. That such flexibility is possible has also been observed by Shiffrin and Schneider (1977), who stated that "the subject engages in the minimal controlled processing necessary to satisfy the task requirements" (p. 154). Recently, a similar account has been presented by Meyer and Kieras (1997a, 1997b) for explaining dual-task performance. They introduced a class of so-called adaptive executive control models. The assumption is that participants can decide to lock out and unlock processes for the different tasks in a rather flexible way by using various scheduling strategies depending on relative task priorities, learning state and so forth (see also Schumacher et al., 1999). Together, these accounts and the supporting results suggest that participants have more attentional control and flexibility, even during the execution of simple tasks, than assumed by some researchers.

\section{Conclusion}

The present article shows that residual shift costs usually observed in task-shift experiments are not only due to passive processes such as interference, as is widely assumed. Rather, the major portion results from attentional control that is required during task execution to guarantee a successful performance. Consequently, the dichotomy between task preparation and task execution does not correspond to the dichotomy between supervisory and passive or exogenous control.

\section{References}

Allport, A., Styles, E. A., \& Hsieh, S. (1994). Shifting intentional set: Exploring the dynamic control of tasks. In C. Umiltà \& M. Moscovitch
(Eds.), Attention and performance XV (pp. 421-452). Cambridge, MA: MIT Press.

Baddeley, A. (1986). Working memory. Oxford, England: Oxford University Press.

Cohen, J. D., Dunbar, K., \& McClelland, J. L. (1990). On the control of automatic processes: A parallel distributed processing account of the Stroop effect. Psychological Review, 97, 177-188.

Cohen, J. D. \& Huston, T. H. (1994). Progress in the use of interactive models for understanding attention and performance. In C. Umiltà \& M. Moscovitch (Eds.), Attention and performance XV. (pp. 453-476). Cambridge, MA: MIT Press.

Dixon, P. (1981). Algorithms and selective attention. Memory \& Cognition, 9, 177-184.

Dixon, P., \& Just, M. A. (1986). A chronometric analysis of strategy preparation in choice reaction time. Memory \& Cognition, 14, 488-500.

Egeth, H. E., \& Yantis, S. (1997). Visual attention: Control, representation, and time course. Annual Review of Psychology, 48, 269-297.

Hübner, R. (1997). The effect of spatial frequency on global precedence and hemispheric differences. Perception \& Psychophysics, 59, 187-201.

Hübner, R. (1998). Hemispheric differences in local-global processing revealed by same-different judgements. Visual Cognition, 5, 457-478.

Hübner, R. (2000). Attention shifting between global and local target levels: The persistence of level-repetition effects. Visual Cognition, 7 , 456-484.

Hübner, R. \& Backer, G. (1999). Perceiving spatially inseparable objects: Evidence for feature-based object selection not mediated by location. Journal of Experimental Psychology: Human Perception and Performance, 25, 1556-1567.

Jersild, A. T. (1927). Mental set and shift. Archives of Psychology, 14, (No. 89 81).

Johnston, W. A. \& Dark, V. J. (1986). Selective attention. Annual Review of Psychology, 37, 43-75.

Kanne, S. M., Balota, D. A. Spieler, D. H \& Faust, M. E. (1998). Explorations of Cohen, Dunbar, and McClelland's (1990) connectionist model of Stroop performance. Psychological Review, 105, 174-187.

Kinchla, R. A. (1992). Attention. Annual Review of Psychology, 43, 711742.

Lamb, M. R., London, B., Pond, H. M., \& Whitt, K. A. (1998). Automatic and controlled processes in the analysis of hierarchical structure. Psychological Science, 9, 14-19.

Logan, G. D. (1985). Executive control of thought and action. Acta Psychologica, 60, 193-210.

Meiran, N. (1996). Reconfiguration of processing mode prior to task performance. Journal of Experimental Psychology: Learning, Memory and Cognition, 22, 1423-1442.

Mewhort, D. J. K., Braun, J. G. \& Heathcote, A. (1992). Response time distributions and the Stroop task: A test of Cohen, Dunbar, and McClelland's (1990) model. Journal of Experimental Psychology: Human Perception and Performance, 18, 872-882.

Meyer, D.E. \& Kieras, D.E. (1997a). A computational theory of executive cognitive processes and multiple-task performance: Part 1. Basic mechanisms. Psychological Review, 104, 3-65.

Meyer, D.E. \& Kieras, D.E. (1997b). A computational theory of executive cognitive processes and multiple-task performance: Part 2. Accounts of psychological refractory-period phenomena. Psychological Review, 104, 749-791.

Monsell, S. (1996). Control of mental processes. In V. Bruce (Ed.), Unsolved mysteries of the mind: Tutorial essays in cognition (pp. 93148). Hove, England: Erlbaum

Navon, D. (1977). Forest before trees: The precedence of global features in visual perception. Cognitive Psychology, 9, 353-383.

Norman, D. A. \& Shallice, T. (1986). Attention to action: Willed and automatic control of behavior. In R.J. Davidson, G.E. Schwartz \& D. 
Shapiro (Eds.), Consciousness and self-regulation (Vol. 4, pp. 1-18) New York: Plenum.

Robertson, L. C. (1996). Attentional persistence for features of hierarchical patterns. Journal of Experimental Psychology: General, 125, 227-249.

Rogers, R. D., \& Monsell, S. (1995). Costs of a predictable switch between simple cognitive tasks. Journal of Experimental Psychology: General, 124, 207-231.

Rubinstein, J., Meyer, D., \& Evans, J. (in press). Executive control of cognitive processes in task switching. Journal of Experimental Psychology: Human Perception and Performance.

Schneider, W., \& Shiffrin, R. M. (1977). Controlled and automatic human information processing: I. Detection, search, and attention. Psychological Review, 84, 1-66.

Schumacher, E. H., Lauber, E. J., Glass, J. M., Zurbriggen, E L., Gmeindl, L., Kieras, D. E. \& Meyer, D. E. (1999). Concurrent response-selection processes in dual-task performance: Evidence for adaptive executive control of task scheduling. Journal of Experimental Psychology: Human Perception and Performance, 25, 791-814.

Shallice, T. (1994). Multiple levels of control processes. In C. Umiltà \& M. Moscovitch (Eds.). Attention and performance XV (pp. 395-420). Cambridge, MA: MIT Press.
Shallice, T. \& Burgess, P. (1996). The domain of supervisory processes and temporal organization of behavior. Philosophical Transactions of the Royal Society of London Series B, 351, 1405-1412.

Shiffrin, R. M., \& Schneider, W. (1977). Controlled and automatic human information processing: II. Perceptual learning, automatic attending, and a general theory. Psychological Review, 84, 127-190.

Spector, A. \& Biederman, I. (1976). Mental set and mental shift revisited. American Journal of Psychology, 89, 669-679.

Sudevan, P. \& Taylor, D. A. (1987). The cuing and priming of cognitive operations. Journal of Experimental Psychology: Human Perception and Performance, 13, 89-103.

Ward, L. M. (1982). Determinants of attention to local and global features of visual forms. Journal of Experimental Psychology: Human Perception and Performance, 8, 562-581. 